Setting First division Belgian (discovery cohort) and UK (replication cohort) soccer.

Patients (or Participants) Players had to be adult out-field players, who did not sustain a HSI in the 6 months before the scan. In total, 96 participants finished the study.

Interventions (or Assessment of Risk Factors) The muscle fibre typology was non-invasively estimated using proton magnetic resonance spectroscopy. During the 1-3 years follow-up period, all sustained injuries were provided by the medical team.

Main Outcome Measurements A cox regression model was used to identify if muscle fibre typology is a risk factor to sustain a HSI. Receiver operating characteristic (ROC) curves were analysed.

Results Belgian players with a fast typology display a 6.7-fold higher risk than slow typology players in experiencing a new HSI (14 HSI; $\mathrm{p}=0.024)$. This was independently confirmed in the UK cohort $(5.0$-fold risk; 13 HSI; $\mathrm{p}=0.023)$. ROC curve analysis revealed an AUC of 0.63 in the total cohort and 0.76 in the discovery cohort, indicating poor to fair sensitivity and specificity.

Conclusions The muscle fibre typology is discovered as a novel and important risk factor to sustain a HSI in professional soccer. This opens a new perspective on the mechanism of HSI, possibly related to more pronounced muscle fatigue in fast typology players. Future personalised prevention strategies could take these interindividual muscle composition differences into account.

\section{HIGHER NECK STRENGTH MAY LOWER HEAD ACCELERATION DURING PURPOSEFUL HEADING IN FOOTBALL: A SYSTEMATIC REVIEW}

Kerry Peek, James M Elliott, Rhonda Orr. Faculty of Health Sciences, The University of Sydney, Sydney, Australia

10.1136/bjsports-2021-IOC.32

Background The long-term effect of repeated head impacts during purposeful heading in football is largely unknown. However, evidence suggests that head acceleration during purposeful heading may be reduced by players having strong neck musculature.

Objective To investigate the relationship between neck strength and head acceleration during purposeful heading in football.

Design This systematic review included a comprehensive search of five electronic databases: EMBASE, MEDLINE, CINAHL, SportsDiscus and Web of Science.

Setting All competitive levels of football regardless of setting (high school, football club or academy) were included.

Patients (or Participants) All football players regardless of age, sex, health status or playing level were included.

Interventions (or Assessment of Risk Factors) Only original research reporting on the relationship between neck strength and head acceleration during purposeful heading in football were included. For experimental research designs, studies were included if neck strengthening was the intervention of interest. Main Outcome Measurements Neck strength measurement using hand-held dynamometry and head acceleration captured during purposeful heading using accelerometry or video analysis.
Results From an initial search of 1174 potentially eligible papers, five cross-sectional studies met the eligibility criteria for inclusion in this review. A total of 190 participants $(67 \%$ Female) with a mean age of 19 years (range 15-24 years) were recruited across the five studies. Playing level ranged from high school football to division I and II collegiate level. Evidence from four level IV studies supported a statistically significant relationship between higher neck strength and lower head acceleration during purposeful heading in football $(p=<0.05 ; r<-0.5)$. The fifth study showed a weak association $(\mathrm{r}=-0.10)$.

Conclusions Data available from cross-sectional research supports that higher neck strength may lower head acceleration during purposeful heading in football. Further evidence is required to determine the most effective method to strengthen the neck musculature in football players and the impact this may have on the long-term health of players.

\section{CAN WE PREDICT RECOVERY AND RE-INJURY FOLLOWING CALF MUSCLE STRAIN INJURY?}

${ }^{1}$ Brady Green, ${ }^{2}$ Monica Lin, ${ }^{1}$ Anthony Schache, ${ }^{1} J o d i e ~ M c C l e l l a n d, ~{ }^{1} A d a m$ Semciw, ${ }^{2}$ Andrew Rotstein, ${ }^{1}$ Jill Cook, ${ }^{1}$ Tania Pizzari. ' La Trobe Sport and Exercise Medicine Research Centre, Melbourne, Australia; ${ }^{2}$ Victoria House Imaging, Melbourne, Australia

\subsection{6/bjsports-2021-IOC.33}

Background Calf muscle strain injuries (CMSI) are prevalent in sport, but investigations into the variable recovery periods and re-injury are lacking.

Objective To determine whether clinical and MRI findings are predictive of recovery and re-injury following CMSI.

Design Retrospective audit of injury data (2014-2017).

Setting Data from the Soft Tissue injury Registry of the Australian Football League (STRAFL).

Patients (or Participants) 149 Australian Football League players.

Interventions (or Assessment of Risk Factors) Blinded radiologists assessed MRI characteristics including: the anatomical location of injury, waviness, and severity of aponeurotic disruption (AD): none (0\%), mild (<50\%), severe (50\%-99\%), complete $(100 \%)$. Clinical factors included: age, mechanism, and injury history.

Main Outcome Measurements The recovery period consisted of four milestones: pain free walking, running at $>90 \%$ of maximum speed, return to full training, return to play (RTP). Risk factors for re-injury were evaluated for early re-injury ( $<2$ months), and overall ( $<2$ seasons). Multi-variable regression was used to evaluate clinical and MRI predictors of recovery and re-injury.

Results 149 CMSI (114 index, 35 re-injuries) were included: 126 soleus, 17 gastrocnemius, and 6 other. The anatomical location of injury did not impact recovery (all, $\mathrm{p}>0.05$ ). CMSI with severe AD took on average 11.9 days longer to RTP than injuries with no $\mathrm{AD}(\mathrm{p}=0.003)$, and the presence of $\operatorname{AD}(p=0.03)$ and a running-related mechanism of injury $(p=0.01)$ were the strongest predictors of a longer RTP for soleus injuries. Older age $(p=0.001)$ and a history of a previous ankle injury $(p=0.03)$ were risk factors for early reinjury, while older age $(p=0.01)$ and a history of previous CMSI $(p=0.002)$ were risk factors for re-injury overall. 